\title{
The efficacy of biochemical markers in patients with ossification of posterior longitudinal ligament of the spine
}

\author{
C Ishihara ${ }^{1}$, K Kushida $^{1}$, M Takahashi*, ${ }^{*}$, T Ohishi ${ }^{1}$, H Murata $^{1}$, A Nagano ${ }^{1}$ and S Goto ${ }^{2}$ \\ ${ }^{1}$ Department of Orthopedic Surgery, Hamamatsu University School of Medicine, Hamamatsu, Japan; ${ }^{2}$ Department of \\ Orthopedic Surgery, Chiba University, 1-8-1 Ibana, Chuoku, Chiba, Japan
}

\begin{abstract}
Study design: Serum levels of carboxyterminal propeptide of type I collagen (PICP), osteocalcin (OC), carboxyterminal telopeptide of type I collagen (ICTP) and urinary levels of pyridinoline (Pyr) and deoxypyridinoline (Dpyr) were measured in patients with ossification of posterior longitudinal ligament of the spine (OPLL) and age-matched control subjects.

Objectives: To evaluate the efficacy of these biochemical markers of the patients with OPLL. Setting: Department of Orthopedic Surgery, Hamamatsu University School of Medicine, Hamamatsu, Japan.

Methods: Spot urine and blood samples were obtained from 20 healthy males aged 45-78 $($ mean $\pm \mathrm{SD} ; 63.0 \pm 11.5)$ and 22 male patients with OPLL aged $46-77$ (mean $\pm \mathrm{SD} ; 59.9 \pm 8.8$ ), and serum levels of PICP, OC, ICTP and urinary levels of Pyr and Dpyr were measured.

Results: There were no significant difference in age, serum PICP, OC, ICTP, urinary Pyr and Dpyr levels between OPLL and control group.

Conclusion: Neither bone formation nor bone resorption was accelerated in the patients with OPLL.

Spinal Cord (2000) 38, 211-213
\end{abstract}

Keywords: OPLL; biochemical markers; carboxyterminal propeptide of type I collagen; carboxyterminal telopeptide of type I collagen; osteocalcin; pyridinoline

\section{Introduction}

Various systemic and local factors are speculated to be related to the development of the ossification of posterior longitudinal ligament of the spine (OPLL), however, the causes of OPLL are still unknown. Generalized hyperosteotic tendency or imbalance between systemic bone formation and bone resorption could be associated with the pathogenesis of OPLL.

Recently, several bone specific markers have been developed and employed to evaluate the degrees of bone resorption and bone formation, separately, in various metabolic bone disorders. Carboxyterminal propeptide of type I collagen (PICP) and osteocalcin (OC) are both bone formation markers. However, those two bone formation markers can reflect bone formation in the different aspects. Carboxyterminal propeptide of type I collagen (PICP) is cleaved off by specific peptidase before the maturation of collagen fibers during its synthesis, and is released into the blood stream. Osteocalcin (OC), a noncollagenous bone matrix protein, is synthesized by osteoblasts. PICP can reflect the degree of de novo type I collagen synthesis, and $\mathrm{OC}$ can reflect

*Correspondence: M Takahasi, Department of Orthopedic Surgery, Hamamatsi University School of Medicine, 3600 Handacho, Hamamatsu, 451-31, Japan the osteoblastic activity. Urinary pyridinoline (Pyr) and deoxypyridinoline (Dpyr), which are intermolecular crosslinks in collagen, have been shown to be sensitive markers of bone resorption. Pyr contributes to the stability of collagen fibers, and is distributed mainly in bone, dentine, and cartilage. ${ }^{1}$ Dpyr is an analog of Pyr and reportedly has a greater specificity for bone than does Pyr. ${ }^{2,3}$ The carboxyterminal telopeptide of type I collagen (ICTP), the breakdown product of type I collagen, is also the bone resorption markers. ICTP is currently available for a marker of multiple myeloma or bone metastasis of malignant tumors. ${ }^{4,5}$

In this study, we approached from the aspect of bone turnover to elucidate the pathogenesis of OPLL. Serum levels of PICP, OC, ICTP and urinary levels of Pyr and Dpyr were measured in patients with OPLL and age-matched control subjects, and the efficacy of these biochemical markers in the patients with OPLL was evaluated.

\section{Methods}

Subjects

Spot urine and blood samples were obtained from 20 healthy males aged $45-78($ mean $\pm S D ; 63.0 \pm 11.5)$ and 
22 male patients with OPLL aged 46-77 (mean \pm SD; $59.9 \pm 8.8$ ). Eleven patients with OPLL were diagnosed at the Department of Orthopedic Surgery in Chiba University and 11 patients with OPLL were diagnosed at the Department of Orthopedic Surgery in Hamamatsu University School of Medicine. The diagnosis of OPLL was made if a patient had at least one segmental OPLL at the cervical or thoracic spine level. Disturbances of spinal cord function below the responsible spinal level were presented in all patients. The most common symptoms were muscular atrophy, muscular weakness, paresthesia, spasticity of lower extremities. However patients could walk with or without crutches to the outpatients clinics. All subjects had no previous histories of metabolic bone diseases and were receiving no medications to affect calcium absorption and metabolism.

\section{Biochemical markers}

Urinary pyridinoline (Pyr) and deoxypyridinoline (Dpyr) were measured by high performance liquid chromatography after acid hydrolysis of urine. The method is previously described elsewhere. ${ }^{6}$ Before hydrolysis, urinary creatinine content was enzymatically determined with an aliquot of each urine sample using a clinical chemistry analyzer (CL-20, Shimadzu, Japan). The values of urinary Pyr and Dpyr in urine samples were expressed per mmol of urinary creatinine. In this method, the intra- and inter-assay CV for Pyr were 4.0 and $8.3 \%$, respectively. Those for Dpyr were 4.2 and $9.5 \%$, respectively.

Serum concentration of PICP and ICTP were measured by PICP-radioimmunoassay-kit and ICTPradioimmunoassay-kit. The intra- and inter-assay $\mathrm{CV}$ for PICP were 3 and $5 \%$, respectively. ${ }^{7}$ The intra- and inter-assay CV for ICTP were 3 and $5 \%$, respectively. ${ }^{5}$ Serum OC were measured by OC-immunoradiometric assay-kit. The intra- and inter-assay $\mathrm{CV}$ for $\mathrm{OC}$ were less than 4 and $6 \%$, respectively. ${ }^{8}$

\section{Statistical analysis}

Data was analyzed using a Stat View II program on a Macintosh computer. Differences between groups were determined by Student's $t$-test (unpaired). $P$ values of less than 0.05 were considered significant.

\section{Results}

The levels of biochemical parameters are indicated in Table 1. There were no significant differences in age, serum PICP, OC, ICTP, urinary Pyr and Dpyr levels between OPLL and control group (Table 1).

\section{Discussion}

There have been several reports that investigated bone formation and bone resorption markers in the patients with OPLL. As far as bone resorption markers, our
Table 1 Values of age, serum and urinary biochemical markers in patients with OPLL and control

\begin{tabular}{lccc}
\hline & $\begin{array}{c}\text { OPLL } \\
(\mathrm{n}=22)\end{array}$ & $\begin{array}{c}\text { Control } \\
(\mathrm{n}=20)\end{array}$ & $\mathrm{P}$ \\
\hline Age & $59.9 \pm 8.8$ & $63.0 \pm 11.5$ & $\mathrm{NS}$ \\
PICP $(\mathrm{ng} / \mathrm{ml})$ & $90.4 \pm 39.5$ & $109.8 \pm 34.8$ & $\mathrm{NS}$ \\
OC $(\mathrm{ng} / \mathrm{ml})$ & $4.9 \pm 2.9$ & $4.4 \pm 2.9$ & $\mathrm{NS}$ \\
ICTP $(\mathrm{ng} / \mathrm{ml})$ & $3.8 \pm 2.3$ & $3.2 \pm 1.1$ & $\mathrm{NS}$ \\
Pyr (nmol/mmol creat.) & $34.1 \pm 19.9$ & $32.2 \pm 12.6$ & $\mathrm{NS}$ \\
Dpyr (nmol/mmol creat.) & $6.7 \pm 4.4$ & $4.8 \pm 2.0$ & NS \\
\hline
\end{tabular}

Data was indicated as mean $\pm \mathrm{SD}$; NS, not significant

results are in good agreement with the reports by Ijiri et $a l^{9}{ }^{9}$ According to Ijiri et al, levels of urinary Pyr and Dpyr were not significantly different between OPLL patients and controls. There have been no reports that evaluated the levels of ICTP in the patients with OPLL. From our results, the breakdown to type I collagen was not accelerated in the patients with OPLL.

As far as bone formation markers, it is still controversial whether serum levels of bone formation markers in the OPLL patients are high or low. Matui et al, reported the significant increase of PICP and intact OC. ${ }^{10}$ According to the cell culture study that was taken from the ossification site of the posterior longitudinal ligament of the spine, these cells expressed the osteoblastic phenotypes from the results of the response to the calcium regulating hormones. ${ }^{11}$ On the other hand Imamura et al, reported that there was no significant increase in intact $\mathrm{OC}$ and there was no increase in bone turnover in patients with OPLL. ${ }^{12}$ In our results, there were no significant differences of PICP and OC between OPLL group and control group. Bone formation was not accelerated in this study.

The greater the progression of the degree of OPLL, the greater the increase in bone formation markers. ${ }^{10}$ Taken together with our results, it is suggested that bone formation activity increased at the time of only progressing to ossification in OPLL patients. Unfortunately, we could not compare the biochemical markers according to the degree of OPLL since the number of OPLL cases was too small to be divided into subgroups. A longitudinal study design will be necessary to examine if bone formation markers can predict the progression of the ossification of posterior longitudinal ligaments.

\section{References}

1 Fujimoto D, Moriguchi T, Ishida T, Hayashi H. The structure of pyridinoline, collagen crosslink. Biochem Biophys Res Commun 1987; 87: $52-57$.

2 Ogawa T, Ono T, Tsuda M, Kawanishi Y. A novel fluor in insoluble collagen: a crosslinking molecule in collagen molecule. Biochem Biophys Res Commun 1982; 107: 1252-1257.

3 Eyre DR. Collagen cross-linking aminoacids. Methods Enzymol 1987; 144: $115-139$. 
4 Elomaa I, Virkkunen P, Risteli L, Risteli J. Serum concentration of the cross-linked carboxyterminal telopeptide of type I collagen (ICTP) is a useful prognostic indicator in multiple myeloma. $\mathrm{Br} \mathrm{J}$ Cancer 1992; 66: $337-341$.

5 Kylmala $\mathrm{T}$ et al. Evaluation of the effect of oral clodronate on skeletal metastases with type I collagen metabolites. Eur J Cancer 1993; 29A: $821-825$.

6 Takahashi M et al. Pre-fractionation with cation exchanger for determination of intermolecular crosslinks, pyridinoline and pentsidine, in hydrolysate. J Liq Chromatogr 1993; 16: $1355-$ 1370.

7 Melkko J, Niemi S, Risteli L, Risteli J. Radioimmunoassay of the carboxyterminal propeptide of human type I procollagen. Clin Chem 1990; 36: $1328-1332$.

8 Patrick G et al. Measurement of serum osteocalcin with a humanspecific two-site immunoradiometric assay. $J$ Bone Miner Res 1992; 7: $1389-1398$.
9 Ijiri $\mathrm{K}$ et al, Imamura $\mathrm{T}$, Sakou T. Urinary excretion of pyridinium crosslinks in the patients of OPLL. Investigation Committee Report on the Ossification of the Spinal Ligaments of the Japanese Ministry of Public Health and Welfare 1993; 22 - 24.

10 Matui H, Yudoh K, Tuji H. Significance of levels of type I procollagen peptide and intact osteocalcin and bone mineral density in patients with ossification of the posterior longitudinal ligaments. Calcif Tissue Int 1996; 59: $397-400$.

11 Ishida Y, Kawai S. Effect of bone-seeking hormones on DNA synthesis, cyclic AMP level, and alkaline phosphatase activity in cultured cells from human posterior longitudinal ligament of the spine. J Bone Miner Res 1993; 8: 1291 - 1300.

12 Imamura K et al. Serum Bone Gra Protein (BGP) of patients with ossification of the posterior longitudinal ligament. Investigation Committee Report in the Ossification of the Spinal Ligaments of the Japanese Ministry of Public Health and Welfare 1991; $1202-$ 1203. 